\title{
Quantum Approach to Epistemic Semantics
}

\author{
Giuseppe Sergioli • Roberto Leporini
}

Received: date / Accepted: date

\begin{abstract}
Quantum information has suggested new forms of quantum logic, called quantum computational logics, where meanings of sentences are represented by pieces of quantum information (generally, density operators of some Hilbert spaces), which can be stored and transmitted by means of quantum particles. This approach can be applied to a semantic characterization of epistemic logical operations, which may occur in sentences like "At time $\mathfrak{t}^{\prime}$ Bob knows that at time $\mathfrak{t}$ Alice knows that the spin-value is up". Each epistemic agent (say, Alice, Bob,...) has a characteristic truth-perspective, corresponding to a particular orthonormal basis of the Hilbert space $\mathbb{C}^{2}$. From a physical point of view, a truth-perspective can be associated to an apparatus that allows one to measure a given observable. An important feature that characterizes the knowledge of any agent is the amount of information that is accessible to him/her (technically, a special set of density operators, which also represents the internal memory of the agent in question). One can prove that interesting epistemic operations are special examples of quantum channels, which generally are not unitary. The act of knowing may involve some intrinsic irreversibility due to possible measurement-procedures or to a loss of information about the environment. We also illustrate some relativistic-like effects that arise in the behavior of epistemic agents.
\end{abstract}

Keywords Quantum logic · quantum operations · epistemic structures

PACS PACS 03.67.Lx · PACS 03.67.-a · 02.10.Ab

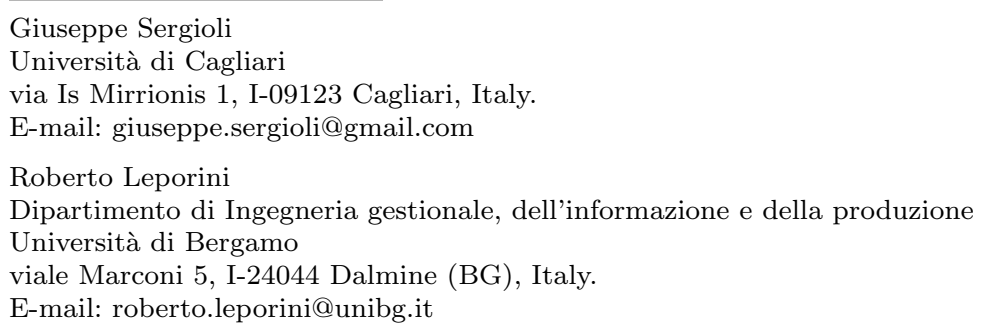




\section{Introduction}

Quantum information has recently suggested new problems and new investigations in logic. An interesting example concerns an abstract theory of epistemic logical operations (like "to know") developed in a Hilbert space environment 1,4. Most standard approaches to epistemic logics have proposed a quite strong characterization of epistemic operations. In such frameworks knowledge is generally modelled as a potential rather than an actual knowledge. Accordingly, a sentence like "Alice knows that the spin-value in the $x$-direction is up" turns out to have the meaning "Alice could know" rather than "Alice actually knows". A consequence of these theories is the unrealistic phenomenon of logical omniscience according to which whenever Alice knows a given sentence, then Alice knows all the logical consequences thereof. Hence, for instance, knowing the axioms of Euclidean geometry should imply knowing all the theorems of the theory.

A more realistic logical theory of knowledge can be developed in the framework of quantum computational logics [6, which are new forms of quantum logic suggested by the theory of quantum logical gates in quantum computation. The basic ideas that underly the semantic characterization of these logics can be sketched as follows. Pieces of quantum information (mathematically represented by density operators of convenient Hilbert spaces) are regarded as possible meanings for the sentences of a given formal language. At the same time, the basic logical connectives of this language are interpreted as particular quantum logical gates: unitary quantum operations that transform density operators in a reversible way. Accordingly, any sentence can be imagined as a synthetic logical description of a quantum circuit.

Is it possible and interesting to describe logical epistemic operations (say, "At time $\mathfrak{t}$ Alice knows") as a special kind of quantum operation? We give a positive answer to this question. The intuitive idea is the following: whenever $\rho$ represents a piece of quantum information (for instance the qubit $|1\rangle$ ), the operation $\mathbf{K}_{\mathfrak{a}_{\mathfrak{t}}}$ (say, "At time $\mathfrak{t}$ Alice knows") transforms $\rho$ into another piece of quantum information $\mathbf{K}_{\mathfrak{a}_{\mathfrak{t}}} \rho$, which lives in the same space of $\rho$ and asserts that "At time $\mathfrak{t}$ Alice knows $\rho$ ". Of course, generally, $\mathbf{K}_{\mathfrak{a}_{\mathfrak{t}}} \rho$ and $\rho$ will be different density operators. On this basis, one can study the behavior of nested epistemic operations, like "At time $\mathfrak{t}^{\prime}$ Bob knows that at time $\mathfrak{t}$ Alice knows $\rho^{\prime \prime}\left(\mathbf{K}_{\mathfrak{b}_{\mathfrak{t}^{\prime}}} \mathbf{K}_{\mathfrak{a}_{\mathfrak{t}}} \rho\right)$.

Like any quantum information, also epistemic pieces of information (say, $\mathbf{K}_{\mathfrak{a}_{\mathfrak{t}}} \rho$ ) may be true or false or indeterminate, where truth-values are defined in terms of a natural notion of quantum probability. Conventionally, one can assume that the two elements $|1\rangle$ and $|0\rangle$ of the canonical orthonormal basis of the Hilbert space $\mathbb{C}^{2}$ represent in this framework the truth-values Truth and Falsity. Accordingly (by application of the Born-rule), the probability of being true for a generic qubit $|\psi\rangle=a_{0}|0\rangle+a_{1}|1\rangle$ will be the number $\mathrm{p}(|\psi\rangle)=\left|a_{1}\right|^{2}$. The definition of Born-like probabilities can be canonically extended to all density operators, living in any product-space $\underbrace{\mathbb{C}^{2} \otimes \ldots \otimes \mathbb{C}^{2}}_{n \text {-times }}$. 
The choice of an orthonormal basis for the space $\mathbb{C}^{2}$ is, obviously, a matter of convention. One can consider infinitely many bases that are determined by the application of a unitary operator $\mathfrak{T}$ to the elements of the canonical basis. From an intuitive point of view, we can think that the operator $\mathfrak{T}$ gives rise to a change of truth-perspective. While the canonical truth-perspective is identified with the pair of bits $|1\rangle$ and $|0\rangle$, in the $\mathfrak{T}$-truth perspective Truth and Falsity are identified with the two qubits $\mathfrak{T}|1\rangle$ and $\mathfrak{T}|0\rangle$, respectively. In this framework, it is not strange to guess that different epistemic agents may have different truth-perspectives, corresponding to different ideas of Truth and Falsity.

From a physical point of view, each truth-perspective can be naturally regarded as associated to a physical apparatus that allows one to measure a given observable. As an example, consider a source emitting a pair of photons correlated in polarization in such a way that both photons have the same polarization (say, the horizontal polarization $|H\rangle$ or the vertical polarization $|V\rangle)$. Suppose that the two photons are in the entangled state

$$
|\psi\rangle=a|H, H\rangle+b|V, V\rangle
$$

(with $|a|^{2}+|b|^{2}=1$ ). The orthonormal basis $B=\{|H\rangle,|V\rangle\}$ represents here a particular truth-perspective. Let us refer to an observer equipped with a polarizer that detects $45^{\circ}$ and $135^{\circ}$ polarized photons. The same state $|\psi\rangle$ will be represented by the observer from a different truth-perspective, corresponding to the orthonormal basis $B^{\prime}=\{|45\rangle,|135\rangle\}$. In fact, by applying the transformation

$$
|H\rangle=\frac{1}{\sqrt{2}}(|45\rangle-|135\rangle) ;|V\rangle=\frac{1}{\sqrt{2}}(|45\rangle+|135\rangle),
$$

the observer will describe the state $|\psi\rangle$ as

$$
|\psi\rangle=\frac{a+b}{2}(|45,45\rangle+|135,135\rangle)-\frac{a-b}{2}(|45,135\rangle+|135,45\rangle) .
$$

Notice that a truth-perspective change gives rise to a different description of one and the same physical state $|\psi\rangle$.

An important feature that characterizes the knowledge of any agent is represented by the amount of information that is accessible to him/her. Technically, the epistemic domain of an agent can be identified with a special set of density operators. From an intuitive point of view, this set can be regarded as the set of pieces of information that our agent is able to understand and to memorize. And the limits of epistemic domains can be used to avoid the unpleasant phenomenon of logical omniscience. One can prove that epistemic operations are not generally unitary. From an intuitive point of view, the act of knowing seems to involve some intrinsic irreversibility due to possible measurement-procedures or to a loss of information about the environment.

In Section 4 we will see how quantum channels are deeply connected with epistemic operations: special examples of irreversible quantum operations that can be used to reach the information stored by density operators. Finally we 
will also illustrate some relativistic-like effects that arise in the behavior of epistemic agents.

An abstract study of epistemic operations in a Hilbert-space environment may have a double interest:

1. from a logical point of view such analysis shows how "thinking in a quantumtheoretic way" can contribute to overcome some crucial difficulties of standard epistemic logics.

2. From a physical point of view this analysis stimulates further investigations about possible correlations between the irreversibility of quantum operations and the kind of "jumps" that seem to characterize acts of knowledge, both in the case of human and of artificial intelligence.

\section{Truth-perspectives and quantum logical gates}

The general mathematical environment is the $n$-fold tensor product of the Hilbert space $\mathbb{C}^{2}$ :

$$
\mathcal{H}^{(n)}:=\underbrace{\mathbb{C}^{2} \otimes \ldots \otimes \mathbb{C}^{2}}_{n \text {-times }},
$$

where all pieces of quantum information live. The elements $|1\rangle=(0,1)$ and $|0\rangle=(1,0)$ of the canonical orthonormal basis $B^{(1)}$ of $\mathbb{C}^{2}$ represent, in this framework, the two classical bits, which can be also regarded as the canonical truth-values Truth and Falsity, respectively. The canonical basis of $\mathcal{H}^{(n)}$ is the set

$$
B^{(n)}=\left\{\left|x_{1}\right\rangle \otimes \ldots \otimes\left|x_{n}\right\rangle:\left|x_{1}\right\rangle, \ldots,\left|x_{n}\right\rangle \in B^{(1)}\right\} .
$$

As usual, we will briefly write $\left|x_{1}, \ldots, x_{n}\right\rangle$ instead of $\left|x_{1}\right\rangle \otimes \ldots \otimes\left|x_{n}\right\rangle$. By definition, a quregister is a unit vector of $\mathcal{H}^{(n)}$. Quregisters thus correspond to pure states, namely to maximal pieces of information about the quantum systems that are supposed to store a given amount of quantum information. We shall also make reference to mixtures of quregisters, represented by density operators $\rho$ of $\mathcal{H}^{(n)}$. Of course, quregisters correspond to special cases of density operators. We will denote by $\mathfrak{D}\left(\mathcal{H}^{(n)}\right)$ the set of all density operators of $\mathcal{H}^{(n)}$, while $\mathfrak{D}=\bigcup_{n}\left\{\mathfrak{D}\left(\mathcal{H}^{(n)}\right)\right\}$ will represent the set of all possible pieces of quantum information.

As observed in the Introduction, from an intuitive point of view, a basischange in $\mathbb{C}^{2}$ can be regarded as a change of truth-perspective. While in the classical case, the truth-values Truth and Falsity are identified with the two classical bits $|1\rangle$ and $|0\rangle$, assuming a different basis corresponds to a different idea of Truth and Falsity. Since any basis-change in $\mathbb{C}^{2}$ is determined by a unitary operator, we can identify a truth-perspective with a unitary operator $\mathfrak{T}$ of $\mathbb{C}^{2}$. We will write:

$$
\left|1_{\mathfrak{T}}\right\rangle=\mathfrak{T}|1\rangle ;\left|0_{\mathfrak{T}}\right\rangle=\mathfrak{T}|0\rangle,
$$

and we will assume that $\left|1_{\mathfrak{T}}\right\rangle$ and $\left|0_{\mathfrak{T}}\right\rangle$ represent, respectively, the truth-values Truth and Falsity of the truth-perspective $\mathfrak{T}$. The canonical truth-perspective 
is, of course, determined by the identity operator I of $\mathbb{C}^{2}$. We will indicate by $B_{\mathfrak{T}}^{(1)}$ the orthonormal basis determined by $\mathfrak{T}$; while $B_{\mathrm{I}}^{(1)}$ will represent the canonical basis.

Any unitary operator $\mathfrak{T}$ of $\mathcal{H}^{(1)}$ can be naturally extended to a unitary operator $\mathfrak{T}^{(n)}$ of $\mathcal{H}^{(n)}$ (for any $n \geq 1$ ):

$$
\mathfrak{T}^{(n)}\left|x_{1}, \ldots, x_{n}\right\rangle=\mathfrak{T}\left|x_{1}\right\rangle \otimes \ldots \otimes \mathfrak{T}\left|x_{n}\right\rangle .
$$

Accordingly, any choice of a unitary operator $\mathfrak{T}$ of $\mathcal{H}^{(1)}$ determines an orthonormal basis $B_{\mathfrak{T}}^{(n)}$ for $\mathcal{H}^{(n)}$ such that:

$$
B_{\mathfrak{T}}^{(n)}=\left\{\mathfrak{T}^{(n)}\left|x_{1}, \ldots, x_{n}\right\rangle:\left|x_{1}, \ldots, x_{n}\right\rangle \in B_{\mathrm{I}}^{(n)}\right\} .
$$

Instead of $\mathfrak{T}^{(n)}\left|x_{1}, \ldots, x_{n}\right\rangle$ we will also write $\left|x_{1_{\mathfrak{T}}}, \ldots, x_{n_{\mathfrak{T}}}\right\rangle$.

The elements of $B_{\mathfrak{T}}^{(1)}$ will be called the $\mathfrak{T}$-bits of $\mathcal{H}^{(1)}$; while the elements of $B_{\mathfrak{T}}^{(n)}$ will represent the $\mathfrak{T}$-registers of $\mathcal{H}^{(n)}$.

On this ground the notions of truth, falsity and probability with respect to any truth-perspective $\mathfrak{T}$ can be defined in a natural way.

Definition 1 (T-true and $\mathfrak{T}$-false registers)

$-\left|x_{1_{\mathfrak{T}}}, \ldots, x_{n_{\mathfrak{T}}}\right\rangle$ is a $\mathfrak{T}$-true register iff $\left|x_{n_{\mathfrak{T}}}\right\rangle=\left|1_{\mathfrak{T}}\right\rangle$;

$-\left|x_{1_{\mathfrak{T}}}, \ldots, x_{n_{\mathfrak{T}}}\right\rangle$ is a $\mathfrak{T}$-false register iff $\left|x_{n_{\mathfrak{T}}}\right\rangle=\left|0_{\mathfrak{T}}\right\rangle$.

In other words, the $\mathfrak{T}$-truth-value of a $\mathfrak{T}$-register (which corresponds to a sequence of $\mathfrak{T}$-bits) is determined by its last element. 1

Definition 2 (T-truth and $\mathfrak{T}$-falsity)

- The $\mathfrak{T}$-truth of $\mathcal{H}^{(n)}$ is the projection operator ${ }^{\mathfrak{T}} P_{1}^{(n)}$ that projects over the closed subspace spanned by the set of all $\mathfrak{T}$ - true registers;

- the $\mathfrak{T}$-falsity of $\mathcal{H}^{(n)}$ is the projection operator ${ }^{\mathfrak{T}} P_{0}^{(n)}$ that projects over the closed subspace spanned by the set of all $\mathfrak{T}$ - false registers.

In this way, truth and falsity are dealt with as mathematical representatives of possible physical properties. Accordingly, by applying the Born-rule, one can naturally define the probability-value of any density operator with respect to the truth-perspective $\mathfrak{T}$.

Definition 3 (T-Probability)

For any $\rho \in \mathfrak{D}\left(\mathcal{H}^{(n)}\right)$,

$$
\mathrm{p}_{\mathfrak{T}}(\rho):=\operatorname{Tr}\left({ }^{\mathfrak{T}} P_{1}^{(n)} \rho\right)
$$

where $\operatorname{Tr}$ is the trace-functional.

\footnotetext{
1 As we will see, the application of a classical reversible gate to a register $\left|x_{1}, \ldots, x_{n}\right\rangle$ transforms the (canonical) bit $\left|x_{n}\right\rangle$ into the target-bit $\left|x_{n}^{\prime}\right\rangle$, which behaves as the final truth-value. This justifies our choice in Definition 1
} 
We interpret $\mathbf{p}_{\mathfrak{T}}(\rho)$ as the probability that the information $\rho$ satisfies the $\mathfrak{T}$ Truth.

In the particular case of qubits, we will obviously obtain:

$$
\mathrm{p}_{\mathfrak{T}}\left(a_{0}\left|0_{\mathfrak{T}}\right\rangle+a_{1}\left|1_{\mathfrak{T}}\right\rangle\right)=\left|a_{1}\right|^{2} .
$$

Two truth-perspectives $\mathfrak{T}_{1}$ and $\mathfrak{T}_{2}$ are called probabilistically equivalent iff for any density operator $\rho, \mathbf{p}_{\mathfrak{T}_{1}}(\rho)=\mathbf{p}_{\mathfrak{T}_{2}}(\rho)$.

For any choice of a truth-perspective $\mathfrak{T}$, the set $\mathfrak{D}$ of all density operators can be pre-ordered by a relation that is defined in terms of the probabilityfunction $\mathrm{p}_{\mathfrak{T}}$.

Definition 4 (Preorder) $\rho \preceq \mathfrak{T} \sigma$ iff $\mathbf{p}_{\mathfrak{T}}(\rho) \leq \mathbf{p}_{\mathfrak{T}}(\sigma)$.

This preorder relation plays an important role in the semantics of quantum computational logics. For, the logical consequence-relation between sentences is defined in terms of $\preceq \mathfrak{T}$ (see Section 4).

As is well known, quantum information is processed by quantum logical gates (briefly, gates): unitary operators that transform quregisters into quregisters in a reversible way.

In this article we will consider some well known quantum gates 77: the negation $\mathrm{NOT}^{(\mathrm{n})}$, the Toffoli gate $\mathrm{T}^{(\mathrm{n}, \mathrm{m}, \mathrm{p})}$, the controlled-not gate $\mathrm{XOR}^{(\mathrm{n}, \mathrm{m})}$, the Hadamard-gate $\sqrt{\mathrm{I}}^{(n)}$ and the square root of the negation $\sqrt{\mathrm{NOT}}^{(n)}$, that play a special role both from the computational and from the logical point of view.

All gates can be naturally transposed from the canonical truth-perspective to any truth-perspective $\mathfrak{T}$. Let $G^{(n)}$ be any gate defined with respect to the canonical truth-perspective. The twin-gate $G_{\mathfrak{T}}^{(n)}$, defined with respect to the truth-perspective $\mathfrak{T}$, is determined as follows:

$$
G_{\mathfrak{T}}^{(n)}:=\mathfrak{T}^{(n)} G^{(n)} \mathfrak{T}^{(n) \dagger},
$$

where $\mathfrak{T}^{\dagger}$ is the adjoint of $\mathfrak{T}$.

All $\mathfrak{T}$-gates, defined on $\mathcal{H}^{(n)}$, can be canonically extended to the set of all density operators of $\mathcal{H}^{(n)}$. Let $G_{\mathfrak{T}}$ be any gate defined on $\mathcal{H}^{(n)}$. The corresponding unitary quantum operation ${ }^{\mathfrak{D}} G_{\mathfrak{T}}$ is defined as follows for any $\rho \in \mathfrak{D}\left(\mathcal{H}^{(n)}\right)$ :

$$
{ }^{\mathfrak{D}} G_{\mathfrak{T}} \rho=G_{\mathfrak{T}} \rho G_{\mathfrak{T}}^{\dagger} .
$$

It is interesting to consider a convenient notion of distance between truthperspectives. As is well known, different definitions of distance between vectors can be found in the literature. For our aims it is convenient to adopt the Fubini-Study definition of distance between two qubits.

Definition 5 (The Fubini-Study distance)

Let $|\psi\rangle$ and $|\varphi\rangle$ be two qubits.

$$
d(|\psi\rangle,|\varphi\rangle)=\frac{2}{\pi} \arccos |\langle\psi \mid \varphi\rangle| .
$$


This notion of distance satisfies the following conditions:

1. $d(|\psi\rangle,|\varphi\rangle)$ is a metric distance;

2. $|\psi\rangle \perp|\varphi\rangle \Rightarrow d(|\psi\rangle,|\varphi\rangle)=1$;

3. $d\left(|1\rangle,\left|1_{\text {Bell }}\right\rangle\right)=\frac{1}{2}$, where $|1\rangle$ is the canonical truth, while $\left|1_{\text {Bell }}\right\rangle=\sqrt{\mathrm{I}}^{(1)}|1\rangle=$ $\left(\frac{1}{\sqrt{2}},-\frac{1}{\sqrt{2}}\right)$ represents the Bell-truth (which corresponds to a maximal uncertainty with respect to the canonical truth).

On this ground, one can naturally define the epistemic distance between two truth-perspectives.

Definition 6 (Epistemic distance)

Let $\mathfrak{T}_{1}$ and $\mathfrak{T}_{2}$ be two truth-perspectives.

$$
d^{E p}\left(\mathfrak{T}_{1}, \mathfrak{T}_{2}\right)=d\left(\left|1_{\mathfrak{T}_{1}}\right\rangle,\left|1_{\mathfrak{T}_{2}}\right\rangle\right) .
$$

In other words, the epistemic distance between the truth-perspectives $\mathfrak{T}_{1}$ and $\mathfrak{T}_{2}$ is identified with the distance between the two qubits that represent the truth-value Truth in $\mathfrak{T}_{1}$ and in $\mathfrak{T}_{2}$, respectively.

\section{Epistemic operations and epistemic structures}

We will now introduce the concepts of (logical) epistemic operation and of epistemic structure.

Definition 7 ((Logical) epistemic operation and strong epistemic operation) A (logical) epistemic operation of the space $\mathcal{H}^{(n)}$ with respect to the truthperspective $\mathfrak{T}$ is a map

$$
\mathbf{E}_{\mathfrak{T}}^{(n)}: \mathcal{B}\left(\mathcal{H}^{(n)}\right) \mapsto \mathcal{B}\left(\mathcal{H}^{(n)}\right),
$$

where $\mathcal{B}\left(\mathcal{H}^{(n)}\right)$ is the set of all bounded operators of $\mathcal{H}^{(n)}$. The following conditions are required:

1. $\mathbf{E}_{\mathfrak{T}}^{(n)}$ is associated with an epistemic domain $\operatorname{EpD}\left(\mathbf{E}_{\mathfrak{T}}^{(n)}\right)$, which is a subset of $\mathfrak{D}\left(\mathcal{H}^{(n)}\right)$;

2. for any $\rho \in \mathfrak{D}\left(\mathcal{H}^{(n)}\right), \mathbf{E}_{\mathfrak{T}}^{(n)} \rho \in \mathfrak{D}\left(\mathcal{H}^{(n)}\right)$;

3. $\forall \rho \in \mathfrak{D}\left(\mathcal{H}^{(n)}\right): \rho \notin E p D\left(\mathbf{E}_{\mathfrak{T}}^{(n)}\right) \Rightarrow \mathbf{E}_{\mathfrak{T}}^{(n)} \rho=\overline{\rho_{0}}$ (where $\overline{\rho_{0}}$ is a fixed density operator of $\left.\mathfrak{D}\left(\mathcal{H}^{(n)}\right)\right)$.

An epistemic operation $\mathbf{E}_{\mathfrak{T}}^{(n)}$ is called strong iff $\mathbf{E}_{\mathfrak{T}}^{(n)} \rho \preceq \mathfrak{T} \rho$, for any $\rho \in$ $\operatorname{EpD}\left(\mathbf{E}_{\mathfrak{T}}\right)$ (where $\preceq_{\mathfrak{T}}$ is the preorder relation defined by Def 4 ).

As expected, an intuitive interpretation of $\mathbf{E}_{\mathfrak{T}}^{(n)} \rho$ is the following: "the piece of information $\rho$ is known/believed". The strong epistemic operation described by $\mathbf{E}_{\mathfrak{T}}^{(n)}$ is limited by a given epistemic domain (which is intended to represent the information accessible to a given agent, or also his/her memory, relatively to the space $\left.\mathcal{H}^{(n)}\right)$. Whenever a piece of information $\rho$ does not belong to 
the epistemic domain of $\mathbf{E}_{\mathfrak{T}}^{(n)}$, then $\mathbf{E}_{\mathfrak{T}}^{(n)} \rho$ collapses into a fixed element $\overline{\rho_{0}}$ (which may be identified, for instance, with the maximally uncertain information $\frac{1}{2^{n}} \mathrm{I}^{(n)}$ or with the $\mathfrak{T}$-Falsity $\left.\frac{1}{2^{n-1}}{ }^{\mathfrak{T}} P_{0}^{(n)}\right)$. At the same time, whenever $\rho$ belongs to the epistemic domain of $\mathbf{E}_{\mathfrak{T}}^{(n)}$, it seems reasonable to assume that the probability-values of $\rho$ and $\mathbf{E}_{\mathfrak{T}}^{(n)} \rho$ are correlated: the probability of the quantum information asserting that " $\rho$ is known/believed" should always be less than or equal to the probability of $\rho$. Hence, in particular, we have:

$$
\operatorname{p}_{\mathfrak{T}}\left(\mathbf{E}_{\mathfrak{T}}^{(n)} \rho\right)=1 \Rightarrow \mathrm{p}_{\mathfrak{T}}(\rho)=1 .
$$

But generally, not the other way around! In other words, pieces of quantum information that are certainly known are certainly true (with respect to the truth-perspective in question).

A strong epistemic operation $\mathbf{E}_{\mathfrak{T}}^{(n)}$ is called non-trivial iff for at least one density operator $\rho \in E p D\left(\mathbf{E}_{\mathfrak{T}}^{(n)}\right), \mathbf{p}_{\mathfrak{T}}\left(\mathbf{E}_{\mathfrak{T}}^{(n)} \rho\right)<\mathbf{p}_{\mathfrak{T}}(\rho)$. Notice that strong epistemic operations do not generally preserve pure states [1,2,3].

One can prove that non-trivial epistemic operations cannot be represented by unitary quantum operations, being generally irreversible [1]. Their behavior is, in a sense, similar to the behavior of measurement-operations.

At the same time, some interesting epistemic operations can be represented by the more general notion of quantum channel defined below. ${ }^{2}$

Definition 8 (Quantum channel) ${ }^{3}$

A quantum channel on $\mathcal{H}^{(n)}$ is a linear map $\mathcal{E}^{(n)}$ from $\mathcal{B}\left(\mathcal{H}^{(n)}\right)$ to $\mathcal{B}\left(\mathcal{H}^{(n)}\right)$ such that for some set $I$ of indices there exists a set $\left\{E_{i}\right\}_{i \in I}$ of elements of $\mathcal{B}\left(\mathcal{H}^{(n)}\right)$ satisfying the following conditions:

1. $\sum_{i} E_{i}^{\dagger} E_{i}=\mathrm{I}^{(n)}$;

2. $\forall A \in \mathcal{B}\left(\mathcal{H}^{(n)}\right): \mathcal{E}^{(n)}(A)=\sum_{i} E_{i} A E_{i}^{\dagger}$.

A set $\left\{E_{i}\right\}_{i \in I}$ such that $\sum_{i} E_{i}^{\dagger} E_{i}=\mathrm{I}^{(n)}$ is usually called a system of Kraus operators. One can prove that quantum channels are trace-preserving, and hence transform density operators into density operators.

Of course, unitary quantum operations ${ }^{\mathfrak{D}} G^{(n)}$ are special cases of quantum channels, for which $\left\{E_{i}\right\}_{i \in I}=\left\{G^{(n)}\right\}$. As expected, quantum channels can be defined with respect to any truth-perspective $\mathfrak{T}$.

We define now some possible properties of epistemic operations that have a significant logical interest. One is dealing with strong conditions that are generally violated in the real use of our intuitive notion of knowledge.

\footnotetext{
2 Quantum channels represent particular cases of quantum operations. The concept of quantum operation is a quite general notion that permits us to represent at the same time symmetry transformations of quantum states, effects and measurements. In particular, it has been shown that for open systems, interacting with an environment, the Schrödingerequation should be generalized to a superoperator-equation, describing how an initial pure state evolves into a mixed state, transformation that has the form of a quantum operation. See, for instance, $[5,8$.

3 This definition is based on the so called Kraus first representation theorem. See [9].
} 
Definition 9 (Introspection, consistency and monotonicity)

A strong epistemic operation $\mathbf{E}_{\mathfrak{T}}^{(n)}$ of $\mathcal{H}^{(n)}$ (with respect to the truth-perspective

$\mathfrak{T})$ is called

1. positively introspective iff for any $\rho \in \mathfrak{D}\left(\mathcal{H}^{(n)}\right)$ :

$$
\mathrm{p}_{\mathfrak{T}}\left(\mathbf{E}_{\mathfrak{T}}^{(n)} \rho\right) \leq \mathrm{p}_{\mathfrak{T}}\left(\mathbf{E}_{\mathfrak{T}}^{(n)} \mathbf{E}_{\mathfrak{T}}^{(n)} \rho\right) .
$$

In other words, whenever we know/believe we know/believe that we know/believe.

2. Negatively introspective iff for any $\rho \in \mathfrak{D}\left(\mathcal{H}^{(n)}\right)$ :

$$
\mathrm{p}_{\mathfrak{T}}\left({ }^{\mathfrak{D}} \mathrm{NOT}_{\mathfrak{T}} \mathbf{E}_{\mathfrak{T}}^{(n)} \rho\right) \leq \mathrm{p}_{\mathfrak{T}}\left(\mathbf{E}_{\mathfrak{T}}^{(n)} \mathfrak{D}^{\mathrm{NOT}} \mathbf{T}_{\mathfrak{T}} \mathbf{E}_{\mathfrak{T}}^{(n)} \rho\right)
$$

In other words, whenever we do not know/believe we know/believe that we do not know/believe.

3. Probabilistically consistent iff for any $\rho \in \mathfrak{D}\left(\mathcal{H}^{(n)}\right)$ :

$$
\mathrm{p}_{\mathfrak{T}}\left(\mathbf{E}_{\mathfrak{T}}^{(n)} \rho\right) \leq \mathrm{p}_{\mathfrak{T}}\left({ }^{\mathfrak{D}} \mathrm{NOT}_{\mathfrak{T}} \mathbf{E}_{\mathfrak{T}}^{(n)} \mathfrak{D} \mathrm{NOT}_{\mathfrak{T}} \rho\right) .
$$

In other words, whenever we know/believe an information we do not know/believe its negation.

4. monotonic iff for any $\rho, \sigma \in \mathfrak{D}\left(\mathcal{H}^{(n)}\right)$ :

$$
\mathbf{p}_{\mathfrak{T}}(\rho) \leq \mathbf{p}_{\mathfrak{T}}(\sigma) \Rightarrow \mathbf{p}_{\mathfrak{T}}\left(\mathbf{E}_{\mathfrak{T}}^{(n)} \rho\right) \leq \mathbf{p}_{\mathfrak{T}}\left(\mathbf{E}_{\mathfrak{T}}^{(n)} \sigma\right) .
$$

Using the concepts defined above, we can finally introduce the notion of epistemic quantum computational structure. From an intuitive point of view, an epistemic quantum computational structure can be described as a system consisting of a set of epistemic agents evolving in time (where time is dealt with as a finite sequence of instants $\left(\mathfrak{t}_{1}, \ldots, \mathfrak{t}_{n}\right)$ ). Each agent $\mathfrak{a}$ is characterized by a truth-perspective $\mathfrak{T}_{\mathfrak{a}}$, which (for the sake of simplicity) is supposed to be constant in time. At any time $\mathfrak{t}$ and for any Hilbert space $\mathcal{H}^{(n)}$, each agent is associated to a strong epistemic operation $\mathbf{E}_{\mathfrak{T}_{\mathfrak{a}}, \mathfrak{a}_{t}}^{(n)}$, whose epistemic domain represents the amount of information that our agent is able to understand and to memorize at that particular time (relatively to the space $\mathcal{H}^{(n)}$ ). When $\rho$ belongs to the epistemic domain of $\mathbf{E}_{\mathfrak{T}_{\mathfrak{a}}}^{(n)}, \mathfrak{a}_{\mathfrak{t}}$, then the number $\mathbf{p}_{\mathfrak{T}_{\mathfrak{a}}}\left(\mathbf{E}_{\mathfrak{T}_{\mathfrak{a}}, \mathfrak{a}_{\mathfrak{t}}}^{(n)} \rho\right)$ represents the probability that agent $\mathfrak{a}$ at time $\mathfrak{t}$ knows/believes the quantum information $\rho$.

Definition 10 (Epistemic quantum computational structure) An epistemic quantum computational structure is a system

$$
\mathcal{S}=(T, A g, \text { TrPersp, Inf, } \mathbf{U}, \mathbf{B}, \mathbf{K}),
$$

where:

1. $T$ is a time-sequence $\left(\mathfrak{t}_{1}, \ldots, \mathfrak{t}_{n}\right)$;

2. $A g$ is a set of epistemic agents where each agent $\mathfrak{a}$ is represented as a function of the time $\mathfrak{t}$ in $T$. We will write $\mathfrak{a}_{\mathfrak{t}}$ instead of $\mathfrak{a}(\mathfrak{t})$; 
3. TrPersp is a map that assigns to any agent $\mathfrak{a}$ a truth-perspective $\mathfrak{T}_{\mathfrak{a}}$ (the truth-perspective of $\mathfrak{a}$ );

4. Inf is a map that assigns to any $\mathfrak{a}_{\mathfrak{t}}$ and to any $n \geq 1$ a map, called (logical) information operation

$$
\operatorname{Inf}_{\mathfrak{T}_{\mathfrak{a}}, \mathfrak{a}_{\mathfrak{t}}}^{(n)}: \mathcal{B}\left(\mathcal{H}^{(n)}\right) \mapsto \mathcal{B}\left(\mathcal{H}^{(n)}\right),
$$

which is an epistemic operation with respect to the truth-perspective $\mathfrak{T}_{\mathfrak{a}}$ (the truth-perspective of agent $\mathfrak{a}$ ).

5. $\mathbf{U}$ is a map that assigns to any $\mathfrak{a}_{\mathfrak{t}}$ and to any $n \geq 1$ a map, called (logical) understandig operation

$$
\mathbf{U}_{\mathfrak{T}_{\mathfrak{a}}, \mathfrak{a}_{\mathfrak{t}}}^{(n)}: \mathcal{B}\left(\mathcal{H}^{(n)}\right) \mapsto \mathcal{B}\left(\mathcal{H}^{(n)}\right),
$$

which is an epistemic operation with respect to the truth-perspective $\mathfrak{T}_{\mathfrak{a}}$.

6. $\mathbf{B}$ is a map that assigns to any $\mathfrak{a}_{\mathfrak{t}}$ and to any $n \geq 1$ a map, called (logical) belief operation

$$
\mathbf{B}_{\mathfrak{T}_{\mathfrak{a}}, \mathfrak{a}_{\mathfrak{t}}}^{(n)}: \mathcal{B}\left(\mathcal{H}^{(n)}\right) \mapsto \mathcal{B}\left(\mathcal{H}^{(n)}\right),
$$

which is a strong epistemic operation with respect to the truth-perspective $\mathfrak{T}_{\mathfrak{a}}$.

7. $\mathbf{K}$ is a map that assigns to any $\mathfrak{a}_{\mathfrak{t}}$ and to any $n \geq 1$ a map, called (logical) knowledge operation

$$
\mathbf{K}_{\mathfrak{T}_{\mathfrak{a}}, \mathfrak{a}_{\mathfrak{t}}}^{(n)}: \mathcal{B}\left(\mathcal{H}^{(n)}\right) \mapsto \mathcal{B}\left(\mathcal{H}^{(n)}\right),
$$

which is a strong epistemic operation with respect to the truth-perspective $\mathfrak{T}_{\mathfrak{a}}$. The following conditions are required:

(i) $\mathbf{K}_{\mathfrak{T}_{\mathfrak{a}}, \mathfrak{a}_{\mathfrak{t}}}^{(n)} \rho \preceq \mathfrak{T}_{\mathfrak{T}_{\mathfrak{a}}}, \mathbf{B}_{\mathfrak{t}}^{(n)} \rho$, for any $\rho \in \operatorname{EpD}\left(\mathbf{K}_{\mathfrak{T}_{\mathfrak{a}}, \mathfrak{a}_{\mathfrak{t}}}^{(n)}\right)$;

(ii) $\mathbf{B}_{\mathfrak{T}_{\mathfrak{a}}, \mathfrak{a}_{\mathfrak{t}}}^{(n)} \rho \preceq \mathfrak{T} \mathbf{U}_{\mathfrak{T}_{\mathfrak{a}}, \mathfrak{a}_{\mathfrak{t}}}^{(n)} \rho$, for any $\rho \in \operatorname{EpD}\left(\mathbf{B}_{\mathfrak{T}_{\mathfrak{a}}, \mathfrak{a}_{\mathfrak{t}}}^{(n)}\right.$ ) (where $\preceq \mathfrak{T}$ is the preorder relation defined by Def 4 ;

(iii) $\operatorname{EpD}\left(\mathbf{K}_{\mathfrak{T}_{\mathfrak{a}}, \mathfrak{a}_{\mathfrak{t}}}^{(n)}\right) \subseteq \operatorname{Ep} D\left(\mathbf{B}_{\mathfrak{T}_{\mathfrak{a}}, \mathfrak{a}_{\mathfrak{t}}}^{(n)}\right) \subseteq \operatorname{Ep} D\left(\mathbf{U}_{\mathfrak{T}_{\mathfrak{a}}, \mathfrak{a}_{\mathfrak{t}}}^{(n)}\right) \subseteq \operatorname{EpD}\left(\operatorname{Inf}_{\mathfrak{T}_{\mathfrak{a}}, \mathfrak{a}_{\mathfrak{t}}}^{(n)}\right)$.

From an epistemic point of view, an agent can be certain to have an uncertain information and vice versa.

\section{Quantum noise channels as epistemic operations}

We will now illustrate some examples of strong epistemic operations that may be interesting from a physical point of view. One is dealing with special cases of quantum noise channels, which can be, generally, obtained from some unitary operators, tracing out the ancillary qubits that describe the environment.

Let $\alpha, \beta, \gamma$ be complex numbers such that $|\alpha|^{2}+|\beta|^{2}+|\gamma|^{2} \leq 1$. Consider the following system of Kraus operators:

$$
\begin{aligned}
& E_{0}=\sqrt{1-|\alpha|^{2}-|\beta|^{2}-|\gamma|^{2}} \mathrm{I} \\
& E_{1}=|\alpha| \sigma_{x} \\
& E_{2}=|\beta| \sigma_{y} \\
& E_{3}=|\gamma| \sigma_{z}
\end{aligned}
$$


(where $\sigma_{x}, \sigma_{y}, \sigma_{z}$ are the three Pauli matrices).

Define ${ }^{\alpha, \beta, \gamma} \mathcal{E}^{(1)}$ as follows for any $\rho \in \mathfrak{D}\left(\mathbb{C}^{2}\right)$ :

$$
{ }_{\alpha, \beta, \gamma} \mathcal{E}^{(1)} \rho=\sum_{i=0}^{3} E_{i} \rho E_{i}^{\dagger} .
$$

We have:

$$
{ }^{\alpha, \beta, \gamma} \mathcal{E}^{(1)} \rho=\left(1-|\alpha|^{2}-|\beta|^{2}-|\gamma|^{2}\right) \rho+|\alpha|^{2} \sigma_{x} \rho \sigma_{x}+|\beta|^{2} \sigma_{y} \rho \sigma_{y}+|\gamma|^{2} \sigma_{z} \rho \sigma_{z}
$$

One can prove that for any choice of $\alpha, \beta, \gamma\left(\right.$ such that $\left.|\alpha|^{2}+|\beta|^{2}+|\gamma|^{2} \leq 1\right)$, the map ${ }^{\alpha, \beta, \gamma} \mathcal{E}^{(1)}$ is a quantum channel of the space $\mathbb{C}^{2}$.

Let us refer to the Bloch-sphere corresponding to $\mathfrak{D}\left(\mathbb{C}^{2}\right)$. Any map ${ }^{\alpha, \beta, \gamma} \mathcal{E}^{(1)}$ induces the following vector-transformation (the sphere is deformed into an ellipsoid centered at the origin):

$$
\left(\begin{array}{c}
x \\
y \\
z
\end{array}\right) \mapsto\left(\begin{array}{c}
\left(1-2|\beta|^{2}-2|\gamma|^{2}\right) x \\
\left(1-2|\alpha|^{2}-2|\gamma|^{2}\right) y \\
\left(1-2|\alpha|^{2}-2|\beta|^{2}\right) z
\end{array}\right)
$$

For particular choices of $\alpha, \beta$ and $\gamma$, one obtains some special cases of quantum channels.

- For $\alpha=\beta=\gamma=0$, one obtains the identity operator.

- For $\beta=\gamma=0$, one obtains the bit-fip channel ${ }^{\alpha} \mathcal{B F}^{(1)}$ that flips the two canonical bits (represented as the projection operators ${ }^{\mathrm{I}} P_{0}^{(1)}$ and ${ }^{\mathrm{I}} P_{1}^{(1)}$ ) with probability $|\alpha|^{2}$ :

$$
\begin{aligned}
& { }^{\mathrm{I}} P_{0}^{(1)} \mapsto\left(1-|\alpha|^{2}\right){ }^{\mathrm{I}} P_{0}^{(1)}+\left(|\alpha|^{2}\right){ }^{\mathrm{I}} P_{1}^{(1)} ; \\
& { }^{\mathrm{I}} P_{1}^{(1)} \mapsto\left(1-|\alpha|^{2}\right){ }^{\mathrm{I}} P_{1}^{(1)}+\left(|\alpha|^{2}\right){ }^{\mathrm{I}} P_{0}^{(1)} .
\end{aligned}
$$

The sphere is mapped into an ellipsoid with $x$ as symmetry-axis

- For $\alpha=\gamma=0$, one obtains the bit-phase-flip channel ${ }^{\beta} \mathcal{B P}^{(1)}$ that flips both bits and phase with probability $|\beta|^{2}$. The sphere is mapped into an ellipsoid with $y$ as symmetry-axis.

- For $\alpha=\beta=0$, one obtains the phase-flip channel ${ }^{\gamma} \mathcal{P} \mathcal{F}^{(1)}$ that flips the phase with probability $|\gamma|^{2}$. The sphere is mapped into an ellipsoid with $z$ as symmetry-axis.

- For $|\alpha|^{2}=|\beta|^{2}=|\gamma|^{2}=\frac{p}{4}$, one obtains the depolarizing channel ${ }^{p} \mathcal{D}^{(1)}$. If $p=1$, the polarization along any direction is equal to 0 . The sphere is contracted by a factor $1-p$ and the center of the sphere is a fixed point.

Another interesting example is the generalized amplitude damping $\mathcal{A D}^{(1)}$ induced by the following system of Kraus-operators:

$$
\begin{aligned}
& E_{0}=\sqrt{\lambda}\left(\begin{array}{cc}
1 & 0 \\
0 & \sqrt{1-p}
\end{array}\right) E_{2}=\sqrt{1-\lambda}\left(\begin{array}{cc}
\sqrt{1-p} & 0 \\
0 & 1
\end{array}\right) \\
& E_{1}=\sqrt{\lambda}\left(\begin{array}{cc}
0 & \sqrt{p} \\
0 & 0
\end{array}\right) \quad E_{3}=\sqrt{1-\lambda}\left(\begin{array}{cc}
0 & 0 \\
\sqrt{p} & 0
\end{array}\right)
\end{aligned}
$$


where $\lambda, p \in[0,1]$.

This channel determines the following transformation on the Bloch-sphere (see Fig. 1):

$$
\left(\begin{array}{l}
x \\
y \\
z
\end{array}\right) \mapsto\left(\begin{array}{l}
\sqrt{1-p} x \\
\sqrt{1-p} y \\
(1-p) z+p(2 \lambda-1)
\end{array}\right)
$$

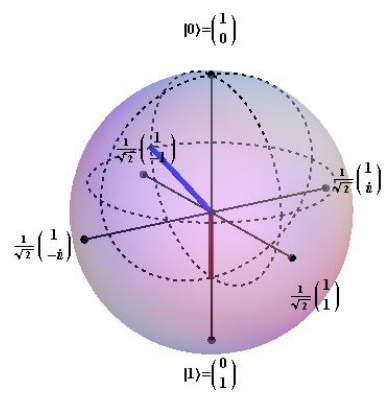

Fig. 1 The amplitude damping channel

The channels we have considered above have been defined with respect to the canonical truth-perspective I. However, as expected, they can be naturally transposed to any truth-perspective $\mathfrak{T}$. Given $\mathcal{E}^{(1)}$ such that $\mathcal{E}^{(1)} \rho=$ $\sum_{i=0}^{3} E_{i} \rho E_{i}^{\dagger}$, the twin-channel $\mathcal{E}_{\mathfrak{T}}^{(1)}$ of $\mathcal{E}^{(1)}$ can be defined as follows:

$$
\mathcal{E}_{\mathfrak{T}}^{(1)} \rho:=\sum_{i} \mathfrak{T} E_{i} \mathfrak{T}^{\dagger} \rho \mathfrak{T} E_{i}^{\dagger} \mathfrak{T}^{\dagger} .
$$

So far we have only considered quantum channels of the space $\mathbb{C}^{2}$. At the same time, any operation $\mathcal{E}_{\mathfrak{T}}^{(1)}$ (defined on $\mathbb{C}^{2}$ ) can be canonically extended to an operation $\mathcal{E}_{\mathfrak{T}}^{(n)}$ defined on the space $\mathcal{H}^{(n)}$ (for any $n>1$ ). Consider a density operator $\rho$ of $\mathcal{H}^{(n)}$ and let $\operatorname{Re}^{n}(\rho)$ represent the reduced state of the $n$-th subsystem of $\rho$. We have: $\operatorname{p}_{\mathfrak{T}}(\rho)=\operatorname{Tr}\left({ }^{\mathfrak{T}} P_{1}^{(1)} \operatorname{Re} d^{n}(\rho)\right)$. In other words, the T-probability of $\rho$ only depends on the T-probability of the $n$-th subsystem of $\rho$. On this basis, it is reasonable to define $\mathcal{E}_{\mathfrak{T}}^{(n)}$ as follows:

$$
\mathcal{E}_{\mathfrak{T}}^{(n)}=\mathrm{I}^{(n-1)} \otimes \mathcal{E}_{\mathfrak{T}}^{(1)} .
$$

Notice that, generally, a quantum channel $\mathcal{E}^{(n)}$ does not represent a strong epistemic operation. We may have, for a $\rho$ (which is supposed to belong to the epistemic domain):

$$
\mathrm{p}_{\mathrm{I}}\left({ }^{\alpha} \mathcal{B F}^{(1)} \rho\right) \not \leq \mathrm{p}_{\mathrm{I}}(\rho),
$$

against the definition of strong epistemic operation.

At the same time, by convenient choices of the epistemic domains, our quantum channels can be transformed into strong epistemic operations. 
Definition 11 (A bit-flip epistemic operation ${ }^{\alpha} \mathbf{K B F}_{\mathfrak{T}}^{(n)}$ )

Let $\alpha \neq 0$. Define ${ }^{\alpha} \mathbf{K B F}_{\mathfrak{T}}^{(n)}$ as follows:

1. $\operatorname{EpD}\left({ }^{\alpha} \mathbf{K B F}_{\mathfrak{T}}^{(n)}\right) \subseteq D=\left\{\rho \in \mathfrak{D}\left(\mathcal{H}^{(n)}\right) \mid \mathbf{p}_{\mathfrak{T}}(\rho) \geq \frac{1}{2}\right\}$.

In other words an agent (whose strong epistemic operation is ${ }^{\alpha} \mathbf{K B F}_{\mathfrak{T}}^{(n)}$ ) only understands pieces of information that are not "too far from the truth".

2. $\rho \in \operatorname{EpD}\left({ }^{\alpha} \mathbf{K} \mathbf{B} \mathbf{F}_{\mathfrak{T}}^{(n)}\right) \Rightarrow{ }^{\alpha} \mathbf{K B F}_{\mathfrak{T}}^{(n)} \rho={ }^{\alpha} \mathcal{B F}_{\mathfrak{T}}^{(n)} \rho$.

Theorem 17

(i) Any ${ }^{\alpha} \mathbf{K B F}_{\mathfrak{T}}^{(n)}$ is a strong epistemic operation. In particular, ${ }^{\alpha} \mathbf{K B F}_{\mathfrak{T}}^{(n)}$ is a non-trivial epistemic operation if there exists at least one $\rho \in \operatorname{EpD}\left({ }^{\alpha} \mathbf{K B F}_{\mathfrak{T}}^{(n)}\right)$ such that $\mathrm{p}_{\mathfrak{T}}(\rho)>\frac{1}{2}$.

(ii) the set $D$ is the maximal set such that the corresponding ${ }^{\alpha} \mathbf{K B F}_{\mathfrak{T}}^{(n)}$ is a strong epistemic operation.

(iii) Let $|\alpha|^{2} \leq \frac{1}{2}$ and let $\operatorname{EpD}\left({ }^{\alpha} \mathbf{K B F}_{\mathfrak{T}}^{(n)}\right)=D$. The following closure property holds: for any $\rho \in D,{ }^{\alpha} \mathbf{K B F}_{\mathfrak{T}}^{(n)} \rho \in D$.

In a similar way one can define strong epistemic operations that correspond to the phase-flip channel, the bit-phase-flip channel, the depolarizing channel and the generalized amplitude-damping channel.

Definition 12 (A phase-flip epistemic operation ${ }^{\gamma} \mathbf{K} \mathbf{P} \mathbf{F}_{\mathfrak{T}}^{(n)}$ )

Let $\gamma \neq 0$. Define ${ }^{\gamma} \mathbf{K} \mathbf{P F} \mathbf{F}_{\mathfrak{T}}^{(n)}$ as follows:

1. $\operatorname{EpD}\left({ }^{\gamma} \mathbf{K} \mathbf{P} \mathbf{F}_{\mathfrak{T}}^{(n)}\right) \subseteq \mathfrak{D}\left(\mathcal{H}^{(n)}\right)$.

2. $\rho \in \operatorname{EpD}\left({ }^{\gamma} \mathbf{K} \mathbf{P} \mathbf{F}_{\mathfrak{T}}^{(n)}\right) \Rightarrow{ }^{\gamma} \mathbf{K} \mathbf{P} \mathbf{F}_{\mathfrak{T}}^{(n)} \rho=\gamma{ }^{\prime} \mathcal{F}_{\mathfrak{T}}^{(n)} \rho$

\section{Theorem 2} $\mathbf{p}_{\mathfrak{T}}\left({ }^{\gamma} \mathbf{K} \mathbf{P} \mathbf{F}_{\mathfrak{T}}^{(n)} \rho\right)=\mathbf{p}_{\mathfrak{T}}(\rho)$, for any $\rho \in \operatorname{EpD}\left({ }^{\gamma} \mathbf{K} \mathbf{P} \mathbf{F}_{\mathfrak{T}}^{(n)}\right)$.

Hence, ${ }^{\gamma} \mathbf{K} \mathbf{P} \mathbf{F}_{\mathfrak{T}}^{(n)}$ is a trivial epistemic operation.

Proof (i)-(ii) Suppose $\rho \in \operatorname{EpD}\left({ }^{\gamma} \mathbf{K} \mathbf{P} \mathbf{F}_{\mathfrak{T}}^{(n)}\right) \subseteq \mathfrak{D}\left(\mathcal{H}^{(n)}\right)$. Let us consider $\mathfrak{T}^{\dagger} \operatorname{Red}^{n}(\rho) \mathfrak{T}=$ $\frac{1}{2}\left(\mathrm{I}+x \sigma_{x}+y \sigma_{y}+z \sigma_{z}\right)$. We have, $\mathbf{p}_{\mathfrak{T}}\left({ }^{\gamma} \mathbf{K} \mathbf{P} \mathbf{F}_{\mathfrak{T}}^{(n)} \rho\right)=\operatorname{Tr}\left({ }^{\mathfrak{T}} P_{1}^{(n)}\right.$

$\left.{ }^{\gamma} \mathbf{K} \mathbf{P} \mathbf{F}_{\mathfrak{T}}^{(n)} \rho\right)=\operatorname{Tr}\left({ }^{\mathrm{I}} P_{1}^{(1)} \sum_{i} E_{i} \mathfrak{T}^{\dagger} \operatorname{Red}^{n}(\rho) \mathfrak{T} E_{i}^{\dagger}\right)=\frac{1-z}{2}=\mathrm{p}_{\mathfrak{T}}(\rho)$.

Definition 13 (A bit-phase-flip epistemic operation ${ }^{\beta} \mathbf{K B P F}_{\mathfrak{T}}^{(n)}$ )

Let $\beta \neq 0$. Define ${ }^{\beta} \mathbf{K B P F}_{\mathfrak{T}}^{(n)}$ as follows:

1. $\operatorname{Ep} D\left({ }^{\beta} \mathbf{K B P F}_{\mathfrak{T}}^{(n)}\right) \subseteq D=\left\{\rho \in \mathfrak{D}\left(\mathcal{H}^{(n)}\right) \mid \mathbf{p}_{\mathfrak{T}}(\rho) \geq \frac{1}{2}\right\}$.

2. $\rho \in \operatorname{EpD}\left({ }^{\beta} \mathbf{K B P F}_{\mathfrak{T}}^{(n)}\right) \Rightarrow{ }^{\beta} \mathbf{K B P F}_{\mathfrak{T}}^{(n)} \rho={ }^{\beta} \mathcal{B} \mathcal{P} \mathcal{F}_{\mathfrak{T}}^{(n)} \rho$.

\section{Theorem 3}

(i) Any ${ }^{\beta} \mathbf{K} \mathbf{B} \mathbf{P} \mathbf{F}_{\mathfrak{T}}^{(n)}$ is a strong epistemic operation. In particular, ${ }^{\beta} \mathbf{K} \mathbf{B} \mathbf{P} \mathbf{F}_{\mathfrak{T}}^{(n)}$ is a non-trivial epistemic operation if there exists at least one $\rho \in \operatorname{EpD}\left({ }^{\beta} \mathbf{K} \mathbf{B P} \mathbf{F}_{\mathfrak{T}}^{(n)}\right)$ such that $\mathrm{p}_{\mathfrak{T}}(\rho)>\frac{1}{2}$. 
(ii) the set $D$ is the maximal set such that the corresponding ${ }^{\beta} \mathbf{K B P F}_{\mathfrak{T}}^{(n)}$ is a strong epistemic operation.

(iii) Let $|\beta|^{2} \leq \frac{1}{2}$ and let $\operatorname{EpD}\left({ }^{\beta} \mathbf{K} \mathbf{B P} \mathbf{F}_{\mathfrak{T}}^{(n)}\right)=D$. The following closure property holds: for any $\rho \in D,{ }^{\alpha} \mathbf{K B F}_{\mathfrak{T}}^{(n)} \rho \in D$.

Proof

Similar to the proof of Theorem 1

Definition 14 (A depolarizing epistemic operation ${ }^{p} \mathbf{K D}_{\mathfrak{T}}^{(n)}$ )

Let $p \neq 0$. Define ${ }^{p} \mathbf{K D}_{\mathfrak{T}}^{(n)}$ as follows:

1. $\operatorname{Ep} D\left({ }^{p} \mathbf{K D}_{\mathfrak{T}}^{(n)}\right) \subseteq D=\left\{\rho \in \mathfrak{D}\left(\mathcal{H}^{(n)}\right) \mid \mathrm{p}_{\mathfrak{T}}(\rho) \geq \frac{1}{2}\right\}$.

2. $\rho \in \operatorname{EpD}\left({ }^{p} \mathbf{K D}_{\mathfrak{T}}^{(n)}\right) \Rightarrow{ }^{p} \mathbf{K D}_{\mathfrak{T}}^{(n)} \rho={ }^{p} \mathcal{D}_{\mathfrak{T}}^{(n)} \rho$.

Notice that for any truth-perspectives $\mathfrak{T},{ }^{p} \mathcal{D}_{\mathfrak{T}}^{(n)}={ }^{p} \mathcal{D}_{\mathrm{I}}^{(n)}$.

\section{Theorem 4}

(i) Any ${ }^{p} \mathbf{K} \mathbf{D}_{\mathfrak{T}}^{(n)}$ is a strong epistemic operation. In particular, ${ }^{p} \mathbf{K} \mathbf{D}_{\mathfrak{T}}^{(n)}$ is a non-trivial epistemic operation if there exists at least one $\rho \in \operatorname{EpD}\left({ }^{p} \mathbf{K D}_{\mathfrak{T}}^{(n)}\right)$ such that $\mathrm{p}_{\mathfrak{T}}(\rho)>\frac{1}{2}$.

(ii) the set $D$ is the maximal set such that the corresponding ${ }^{p} \mathbf{K D}_{\mathfrak{T}}^{(n)}$ is a strong epistemic operation.

(iii) Let $\operatorname{EpD}\left({ }^{p} \mathbf{K} \mathbf{D}_{\mathfrak{T}}^{(n)}\right)=D$. Then, for any $\rho \in \operatorname{EpD}\left({ }^{p} \mathbf{K D}_{\mathfrak{T}}^{(n)}\right)$, we have: ${ }^{p} \mathbf{K D}_{\mathfrak{T}}^{(n)} \rho \in \operatorname{EpD}\left({ }^{p} \mathbf{K D}_{\mathfrak{T}}^{(n)}\right)$.

Proof (i)-(ii) Suppose $\rho \in \operatorname{EpD}\left({ }^{p} \mathbf{K D}_{\mathfrak{T}}^{(n)}\right) \subseteq D$. Let us consider $\mathfrak{T}^{\dagger} \operatorname{Re} d^{n}(\rho) \mathfrak{T}=$ $\frac{1}{2}\left(\mathrm{I}+x \sigma_{x}+y \sigma_{y}+z \sigma_{z}\right)$. We have, $\mathbf{p}_{\mathfrak{T}}\left({ }^{p} \mathbf{K D}_{\mathfrak{T}}^{(n)} \rho\right)=\frac{1-(1-p) z}{2}$. Hence, ${ }^{p} \mathbf{K D}_{\mathfrak{T}}^{(n)} \rho \preceq \mathfrak{T}$ $\rho \Leftrightarrow(1-p) z \geq z \Leftrightarrow z \in[-1,0] \Leftrightarrow \mathbf{p}_{\mathfrak{T}}(\rho) \geq \frac{1}{2}$. Thus, ${ }^{p} \mathbf{K D}_{\mathfrak{T}}^{(n)}$ is an epistemic operation.

(iii) $\operatorname{p}_{\mathfrak{T}}\left({ }^{p} \mathbf{K D}_{\mathfrak{T}}^{(n)} \rho\right)=\frac{1-(1-p) z}{2} \geq \frac{1}{2}$, since $z \in[-1,0]$.

Definition 15 (A generalized amplitude-damping epistemic operation ${ }^{p, \lambda} \mathbf{K A D}_{\mathfrak{T}}^{(n)}$ ) Let $p, \lambda \in[0,1]$. Define ${ }^{p, \lambda} \mathbf{K A D}_{\mathfrak{T}}^{(n)}$ as follows:

1. $\operatorname{EpD}\left({ }^{p, \lambda} \mathbf{K} \mathbf{A D}_{\mathfrak{T}}^{(n)}\right) \subseteq A D=\left\{\rho \in \mathfrak{D}\left(\mathcal{H}^{(n)}\right) \mid \mathbf{p}_{\mathfrak{T}}(\rho) \geq 1-\lambda\right\}$.

2. $\rho \in E p D\left({ }^{p, \lambda} \mathbf{K} \mathbf{A} \mathbf{D}_{\mathfrak{T}}^{(n)}\right) \Rightarrow{ }^{p, \lambda} \mathbf{K A D}_{\mathfrak{T}}^{(n)} \rho={ }^{p, \lambda} \mathcal{A} \mathcal{D}_{\mathfrak{T}}^{(n)} \rho$.

\section{Theorem 5}

(i) Any ${ }^{p, \lambda} \mathbf{K} \mathbf{A} \mathbf{D}_{\mathfrak{T}}^{(n)}$ is a strong epistemic operation. In particular, ${ }^{p, \lambda} \mathbf{K} \mathbf{A D}_{\mathfrak{T}}^{(n)}$ is a non-trivial epistemic operation if there exists at least one $\rho \in \operatorname{EpD}\left({ }^{p, \lambda} \mathbf{K} \mathbf{A D}_{\mathfrak{T}}^{(n)}\right)$ such that $\mathrm{p}_{\mathfrak{T}}(\rho)>1-\lambda$.

(ii) the set $A D$ is the maximal set such that the corresponding ${ }^{p, \lambda} \mathbf{K A D}_{\mathfrak{T}}^{(n)}$ is a strong epistemic operation. 
(iii) Let $\left.\operatorname{EpD}{ }^{p\left({ }^{p} \lambda\right.} \mathbf{K} \mathbf{A} \mathbf{D}_{\mathfrak{T}}^{(n)}\right)=A D$. Then, for any $\rho \in E p D\left({ }^{p, \lambda} \mathbf{K A D}_{\mathfrak{T}}^{(n)}\right)$, we have: ${ }^{p, \lambda} \mathbf{K A D}_{\mathfrak{T}}^{(n)} \rho \in \operatorname{Ep} D\left({ }^{p, \lambda} \mathbf{K} \mathbf{A D}_{\mathfrak{T}}^{(n)}\right)$.

Proof (i)-(ii) Suppose $\rho \in E p D\left({ }^{p, \lambda} \mathbf{K A D}_{\mathfrak{T}}^{(n)}\right) \subseteq A D$. Let us consider $\mathfrak{T}^{\dagger} \operatorname{Red}^{n}(\rho) \mathfrak{T}=$ $\frac{1}{2}\left(\mathrm{I}+x \sigma_{x}+y \sigma_{y}+z \sigma_{z}\right)$. We have, $\mathrm{p}_{\mathfrak{T}}\left({ }^{p, \lambda} \mathbf{K A D}_{\mathfrak{T}}^{(n)} \rho\right)=\frac{1-(1-p) z-p(2 \lambda-1)}{2}$. Hence, ${ }^{p, \lambda} \mathbf{K A D}_{\mathfrak{T}}^{(n)} \rho \preceq_{\mathfrak{T}} \rho \Leftrightarrow(1-p) z+p(2 \lambda-1) \geq z \Leftrightarrow 2 \lambda-1 \geq z \Leftrightarrow \mathrm{p}_{\mathfrak{T}}(\rho) \geq 1-\lambda$. Thus, ${ }^{p, \lambda} \mathbf{K} \mathbf{A D}_{\mathfrak{T}}^{(n)}$ is a strong epistemic operation.

(iii) $\mathrm{p}_{\mathfrak{T}}\left({ }^{p, \lambda} \mathbf{K A D}_{\mathfrak{T}}^{(n)} \rho\right)=\frac{1-(1-p) z-p(2 \lambda-1)}{2} \geq 1-\lambda$, since $2 \lambda-1 \geq z$.

The following theorem sums up some interesting properties of the strong epistemic operations defined above.

\section{Theorem 6}

(i) All strong epistemic operations ${ }^{\alpha} \mathbf{K B F}_{\mathfrak{T}}^{(n)},{ }^{\gamma} \mathbf{K P F}_{\mathfrak{T}}^{(n)},{ }^{\beta} \mathbf{K B P F}_{\mathfrak{T}}^{(n)},{ }^{p} \mathbf{K D}_{\mathfrak{T}}^{(n)}$ are probabilistically consistent.

(ii) All strong epistemic operations ${ }^{p, \lambda} \mathbf{K A D}_{\mathfrak{T}}^{(n)}$ with $\lambda \geq \frac{1}{2}$ are probabilistically consistent.

(iii) All strong epistemic operations ${ }^{\gamma} \mathbf{K} \mathbf{P F}_{\mathfrak{T}}^{(n)}$ are monotonic, positively and negatively introspective.

(iv) All strong epistemic operations ${ }^{\alpha} \mathbf{K B F}_{\mathfrak{T}}^{(n)}$ (with $|\alpha|^{2} \leq \frac{1}{2}$ ), ${ }^{\beta} \mathbf{K} \mathbf{B} \mathbf{P} \mathbf{F}_{\mathfrak{T}}^{(n)}$ (with $|\beta|^{2} \leq \frac{1}{2}$ ), ${ }^{p} \mathbf{K D}_{\mathfrak{T}}^{(n)}$, whose epistemic domain is a subset of $D=$ $\left\{\rho \in \mathfrak{D}\left(\mathcal{H}^{(n)}\right) \mid \mathbf{p}_{\mathfrak{T}}(\rho) \geq \frac{1}{2}\right\}$ are monotonic.

(v) All strong epistemic operations ${ }^{p, \lambda} \mathbf{K} \mathbf{A D}_{\mathfrak{T}}^{(n)}$ whose epistemic domain is a subset of $A D=\left\{\rho \in \mathfrak{D}\left(\mathcal{H}^{(n)}\right) \mid \mathrm{p}_{\mathfrak{T}}(\rho) \geq 1-\lambda\right\}$ are monotonic.

Proof

Let $\mathbf{K}_{\mathfrak{T}}^{(n)} \in\left\{{ }^{\alpha} \mathbf{K B F}_{\mathfrak{T}}^{(n)},{ }^{\gamma} \mathbf{K} \mathbf{P} \mathbf{F}_{\mathfrak{T}}^{(n)},{ }^{\beta} \mathbf{K B P F}_{\mathfrak{T}}^{(n)},{ }^{p} \mathbf{K D}_{\mathfrak{T}}^{(n)}\right\}$ and suppose that $\mathbf{K}_{\mathfrak{T}}^{(n)}$ is induced by the following Kraus operators:

$$
E_{0}=\sqrt{1-|\alpha|^{2}-|\beta|^{2}-|\gamma|^{2}} I ; E_{1}=|\alpha| \sigma_{x} ; E_{2}=|\beta| \sigma_{y} ; E_{3}=|\gamma| \sigma_{z} .
$$

Assume that the fixed element $\overline{\rho_{0}}$ of our epistemic operations is ${ }^{\mathfrak{T}} P_{0}^{(n)}$.

For any $\rho, \rho^{\prime} \in \mathfrak{D}\left(\mathcal{H}^{(n)}\right)$, we can write:

$\mathfrak{T}^{\dagger} \operatorname{Red}^{n}(\rho) \mathfrak{T}=\frac{1}{2}\left(\mathrm{I}+x \sigma_{x}+y \sigma_{y}+z \sigma_{z}\right)$;

$\mathfrak{T}^{\dagger} \operatorname{Red}^{n}\left(\rho^{\prime}\right) \mathfrak{T}=\frac{1}{2}\left(\mathrm{I}+x^{\prime} \sigma_{x}+y^{\prime} \sigma_{y}+z^{\prime} \sigma_{z}\right)$.

(i) Let $\rho \in \mathfrak{D}\left(\mathcal{H}^{(n)}\right)$. We have:

$\rho \notin \operatorname{EpD}\left(\mathbf{K}_{\mathfrak{T}}^{(n)}\right) \Rightarrow \mathrm{p}_{\mathfrak{T}}\left(\mathbf{K}_{\mathfrak{T}}^{(n)} \rho\right)=0$;

${ }^{\mathfrak{D}} \mathrm{NOT}_{\mathfrak{T}} \rho \notin E p D\left(\mathbf{K}_{\mathfrak{T}}^{(n)}\right) \Rightarrow \mathrm{p}_{\mathfrak{T}}\left({ }^{\mathfrak{D}} \mathrm{NOT}_{\mathfrak{T}} \mathbf{K}_{\mathfrak{T}}^{(n)} \mathfrak{D}^{\mathrm{NOT}} \mathrm{T}_{\mathfrak{T}} \rho\right)=1$.

Otherwise, we obtain:

$$
\begin{aligned}
& \mathrm{p}_{\mathfrak{T}}\left({ }^{\mathfrak{D}^{N}} \mathrm{NOT}_{\mathfrak{T}} \mathbf{K}_{\mathfrak{T}}^{(n)} \mathfrak{D}_{\left.\mathrm{NOT}_{\mathfrak{T}} \rho\right)=}\right. \\
& \operatorname{Tr}\left({ }^{\mathrm{I}} P_{1}^{(1)} \mathrm{NOT}^{(1)} \sum_{i}\left(E_{i} \mathrm{NOT}^{(1)} \mathfrak{T}^{\dagger} \operatorname{Red}^{n}(\rho) \mathfrak{T N O T}^{(1)} E_{i}^{\dagger}\right) \mathrm{NOT}^{(1)}\right)=\frac{1-\left(1-2|\alpha|^{2}-2|\beta|^{2}\right) z}{2}= \\
& \mathrm{p}_{\mathfrak{T}}\left(\mathbf{K}_{\mathfrak{T}}^{(n)} \rho\right) .
\end{aligned}
$$

Consequently: $\mathbf{p}_{\mathfrak{T}}\left(\mathbf{K}_{\mathfrak{T}}^{(n)} \rho\right) \leq \mathrm{p}_{\mathfrak{T}}\left({ }^{\mathfrak{D}} \mathrm{NOT}_{\mathfrak{T}} \mathbf{K}_{\mathfrak{T}}^{(n)} \mathfrak{D}_{\mathrm{NOT}} \mathrm{T}_{\mathfrak{T}} \rho\right)$ 
(ii) Similarly.

(iii) By Theorem 2

(iv) Let $\rho, \rho^{\prime} \in \operatorname{EpD}\left(\mathbf{K}_{\mathfrak{T}}^{(n)}\right)$. Suppose that $|\alpha|^{2}+|\beta|^{2}=\frac{1}{2}$. Then we have: $\mathrm{p}_{\mathfrak{T}}\left(\mathbf{K}_{\mathfrak{T}}^{(n)} \rho\right)=\mathrm{p}_{\mathfrak{T}}\left(\mathbf{K}_{\mathfrak{T}}^{(n)} \rho^{\prime}\right)=\frac{1}{2}$. Otherwise we obtain:

$\mathrm{p}_{\mathfrak{T}}\left(\mathbf{K}_{\mathfrak{T}}^{(n)} \rho\right) \leq \mathrm{p}_{\mathfrak{T}}\left(\mathbf{K}_{\mathfrak{T}}^{(n)} \rho^{\prime}\right) \Leftrightarrow \frac{1-\left(1-2|\alpha|^{2}-2|\beta|^{2}\right) z}{2} \leq \frac{1-\left(1-2|\alpha|^{2}-2|\beta|^{2}\right) z^{\prime}}{2} \Leftrightarrow$ $\frac{1-z}{2} \leq \frac{1-z^{\prime}}{2} \Leftrightarrow \mathrm{p}_{\mathfrak{T}}(\rho) \leq \mathrm{p}_{\mathfrak{T}}\left(\rho^{\prime}\right)$.

(v) Similarly.

Notice that in the general case the monotonicity-property can be violated by strong epistemic operations. In fact, the following situation is possible:

$-\rho \preceq \mathfrak{T} \sigma ;$

$-\rho \in E p D\left(\mathbf{K}_{\mathfrak{T}}^{(n)}\right) ; \sigma \notin E p D\left(\mathbf{K}_{\mathfrak{T}}^{(n)}\right) ;$

$-\mathbf{K}_{\mathfrak{T}}^{(n)} \rho \npreceq \mathfrak{T} \mathbf{K}_{\mathfrak{T}}^{(n)} \sigma$.

Generally, the strong epistemic operations ${ }^{\alpha} \mathbf{K B F}_{\mathfrak{T}}^{(n)},{ }^{\beta} \mathbf{K B P F}_{\mathfrak{T}}^{(n)},{ }^{p} \mathbf{K D}_{\mathfrak{T}}^{(n)}$, ${ }^{p, \lambda} \mathbf{K} \mathbf{A} \mathbf{D}_{\mathfrak{T}}^{(n)}$ are neither positively introspective nor negatively introspective. Suppose, for example, that $\operatorname{Ep} D\left(\mathbf{K}_{\mathfrak{T}}^{(n)}\right)=\left\{\mathbf{K}_{\mathfrak{T}}^{(n)}{ }^{\mathfrak{T}} P_{1}^{(n)},{ }^{\mathfrak{T}} P_{1}^{(n)}\right\}$, where

$$
\mathbf{K}_{\mathfrak{T}}^{(n)} \in\left\{{ }^{\alpha} \mathbf{K B F}_{\mathfrak{T}}^{(n)},{ }^{\beta} \mathbf{K B P F}_{\mathfrak{T}}^{(n)},{ }^{p} \mathbf{K D}_{\mathfrak{T}}^{(n)},{ }^{p, \lambda} \mathbf{K A D}_{\mathfrak{T}}^{(n)}\right\} .
$$

By definition of $\mathbf{K}_{\mathfrak{T}}^{(n)}$ we have for any $\rho \in \operatorname{EpD}\left(\mathbf{K}_{\mathfrak{T}}^{(n)}\right): \mathbf{p}_{\mathfrak{T}}\left(\mathbf{K}_{\mathfrak{T}}^{(n)} \rho\right)<\mathbf{p}_{\mathfrak{T}}(\rho)$. Hence, $\mathrm{p}_{\mathfrak{T}}\left(\mathbf{K}_{\mathfrak{T}}^{(n)}{ }_{\mathfrak{T}} P_{1}^{(n)}\right)>\mathrm{p}_{\mathfrak{T}}\left(\mathbf{K}_{\mathfrak{T}}^{(n)} \mathbf{K}_{\mathfrak{T}}^{(n)}{ }_{\mathfrak{T}} P_{1}^{(n)}\right)$. Thus, $\mathbf{K}_{\mathfrak{T}}^{(n)}$ is not positively introspective. Suppose then that $\operatorname{EpD}\left(\mathbf{K}_{\mathfrak{T}}^{(n)}\right)=\left\{{ }^{\mathfrak{D}} \mathrm{NOT}_{\mathfrak{T}} \mathbf{K}_{\mathfrak{T}}^{(n)}{ }_{\mathfrak{T}} P_{0}^{(n)}\right\}$. We have: $\mathrm{p}_{\mathfrak{T}}\left({ }^{\mathfrak{D}} \mathrm{NOT}_{\mathfrak{T}} \mathbf{K}_{\mathfrak{T}}^{(n)}{ }_{\mathfrak{T}} P_{0}^{(n)}\right)>\mathrm{p}_{\mathfrak{T}}\left(\mathbf{K}_{\mathfrak{T}}^{(n)} \mathfrak{D}_{\mathrm{NOT}_{\mathfrak{T}}} \mathbf{K}_{\mathfrak{T}}^{(n)}{ }_{\mathfrak{T}} P_{0}^{(n)}\right)$, where $\mathbf{K}_{\mathfrak{T}}^{(n)} \mathfrak{T} P_{0}^{(n)}={ }^{\mathfrak{T}} P_{0}^{(n)}$. Thus, $\mathbf{K}_{\mathfrak{T}}^{(n)}$ is not negatively introspective.

Truth-perspectives are, in a sense, similar to different frames of reference in relativity. Accordingly, one could try and apply a "relativistic" way of thinking in order to describe how a given agent can "see" the logical behavior of another agent.

As expected, the logical behavior of any agent turns out to depend, in this framework, on two factors:

- his/her idea of Truth and Falsity;

- his/her choice of the gates that correspond to the basic logical operations.

Both these factors are, of course, determined by the agent's truth-perspective T.

As an example let us refer to two agents Alice and $B o b$, whose truthperspectives are $\mathfrak{T}_{\text {Alice }}$ and $\mathfrak{T}_{\text {Bob }}$, respectively. Let $\left\{\left|1_{\text {Alice }}\right\rangle,\left|0_{\text {Alice }}\right\rangle\right\}$ and $\left\{\left|1_{\text {Bob }}\right\rangle\right.$, $\left.\left|0_{B o b}\right\rangle\right\}$ represent the systems of truth-values of our two agents. Furthermore, for any canonical gate ${ }^{\mathfrak{D}} G^{(n)}$ (defined with respect to the canonical truthperspective I), let ${ }^{\mathfrak{D}} G_{\text {Alice }}^{(n)}$ and ${ }^{\mathfrak{D}} G_{\text {Bob }}^{(n)}$ represent the corresponding twin-gates for Alice and for Bob, respectively.

According to the rule assumed in Section 2, we have:

$$
{ }^{\mathfrak{D}} G_{\text {Alice }}^{(n)}={ }^{\mathfrak{D}}\left(\mathfrak{T}_{\text {Alice }}^{(n)} G^{(n)} \mathfrak{T}_{\text {Alice }}^{(n) \dagger}\right) .
$$


In a similar way in the case of Bob.

We will adopt the following conventional terminology.

- When $\left|1_{\text {Bob }}\right\rangle=a_{0}\left|0_{\text {Alice }}\right\rangle+a_{1}\left|1_{\text {Alice }}\right\rangle$, we will say that Alice sees that Bob's Truth is $a_{0}\left|0_{\text {Alice }}\right\rangle+a_{1}\left|1_{\text {Alice }}\right\rangle$. In a similar way, for Bob's Falsity.

- When ${ }^{\mathfrak{D}} G_{\text {Alice }}^{(n)}={ }^{\mathfrak{D}}\left(\mathfrak{T}_{\text {Alice }}^{(n)} G^{(n)} \mathfrak{T}_{\text {Alice }}^{(n) \dagger}\right)$ and ${ }^{\mathfrak{D}} G_{\text {Bob }}^{(n)}={ }^{\mathfrak{D}}\left(\mathfrak{T}_{\text {Bob }}^{(n)} G^{(n)} \mathfrak{T}_{\text {Bob }}^{(n) \dagger}\right)=$ ${ }^{\mathfrak{D}} G_{1_{\text {Alice }}}^{(n)}$ (where ${ }^{\mathfrak{D}} G^{(n)}$ and ${ }^{\mathfrak{D}} G_{1_{\text {Alice }}}^{(n)}$ are canonical gates), we will say that Alice sees Bob using the gate ${ }^{\mathfrak{D}} G_{1_{\text {Alice }}^{(n)}}^{(n)}$ in place of her gate ${ }^{\mathfrak{D}} G_{\text {Alice }}^{(n)}$.

- When ${ }^{\mathfrak{D}} G_{\text {Alice }}^{(n)}={ }^{\mathfrak{D}} G_{\text {Bob }}^{(n)}$ we will say that Alice and Bob see and use the same gate, which represents (in their truth-perspective) the canonical gate $\mathfrak{D}^{(n)}$.

On this basis, one can conclude that, generally, Alice sees a kind of "deformation" in Bob's logical behavior.

In [7] some example have been discussed.

As another insightful example, consider the behavior of the controlled-not gate described by the following theorem.

Theorem 7 Let $\mathfrak{T}_{\mathfrak{a}}$ be the truth-perspective of an agent $\mathfrak{a}$. If ${ }^{\mathfrak{D}} \mathrm{XOR}_{\mathfrak{T}_{\mathfrak{a}}}^{(1,1)}={ }^{\mathfrak{D}} \mathrm{XOR}^{(1,1)}$, then $\mathfrak{T}_{\mathfrak{a}}=e^{i \theta} \mathrm{I}$.

Proof Suppose ${ }^{\mathfrak{D}} \mathrm{XOR}_{\mathfrak{T}_{\mathfrak{a}}}^{(1,1)}={ }^{\mathfrak{D}} \mathrm{XOR}^{(1,1)}$. One can easily show that:

$\left(\mathfrak{T}_{\mathfrak{a}} \otimes \mathfrak{T}_{\mathfrak{a}}\right) \mathrm{XOR}^{(1,1)}=\mathrm{XOR}^{(1,1)}\left(\mathfrak{T}_{\mathfrak{a}} \otimes \mathfrak{T}_{\mathfrak{a}}\right)$. Consequently, by standard algebraic calculations we obtain: $\mathfrak{T}_{\mathfrak{a}}=e^{i \theta} \mathrm{I}$.

As a consequence, one immediately obtains that two agents $\mathfrak{a}$ and $\mathfrak{b}$ can see and use the same XOR-gate only if their truth-perspectives $\mathfrak{T}_{\mathfrak{a}}$ and $\mathfrak{T}_{\mathfrak{b}}$ are probabilistically equivalent.

Moreover, a relativistic way of thinking can also be applied to strong epistemic operations. Alice sees Bob using a phase-flip channel instead of a bit-flip channel as strong epistemic operation. Similarly, some other agent sees Bob's strong epistemic operation acts as a bit-phase-flip channel.

From a logical point of view, examples of epistemic situations as the one we have here investigated, can be formally reconstructed in the framework of a quantum computational semantics (see 4]). Let us briefly recall the basic ideas of this approach. We consider an epistemic quantum computational language $\mathcal{L}^{E p}$ consisting of:

- atomic sentences;

- logical connectives corresponding to the following gates: negation, Toffoli, controlled-not, Hadamard and square root of negation;

- names for epistemic agents (say, Alice, Bob, ...);

- logical epistemic operators, corresponding to (generally irreversible) epistemic operations.

This language can express sentences like "Alice knows that Bob does not know that the spin-value in the $x$-direction is up". The semantics for $\mathcal{L}^{E p}$ provides a convenient notion of model, whose role is assigning informational 
meanings to all sentences. Technically, a model of $\mathcal{L}^{E p}$ is defined as a map Mod that associates to any truth-perspective $\mathfrak{T}$ and to any sentence $\alpha$ a density operator $\rho=\operatorname{Mod}_{\mathfrak{T}}(\alpha)$, living in a Hilbert space $\mathcal{H}^{\alpha}$, whose dimension depends on the linguistic complexity of $\alpha$.

On this basis, one can give a natural definition for the concepts of truth and of logical consequence in terms of the notions of $\mathfrak{T}$-probability $\left(\mathrm{p}_{\mathfrak{T}}\right)$ and of T-preorder $(\preceq \mathfrak{T})$ :

- a sentence $\alpha$ is true with respect to a model Mod and to a truth-perspective $\mathfrak{T}$ (abbreviated as $\left.\vDash_{\operatorname{Mod}, \mathfrak{T}} \alpha\right)$ iff $\mathrm{p}_{\mathfrak{T}}\left(\operatorname{Mod}_{\mathfrak{T}}(\alpha)\right)=1$;

- a sentence $\beta$ is a logical consequence of a sentence $\alpha$ (abbreviated as $\alpha \vDash \beta$ ) iff for any model Mod and any truth-perspective $\mathfrak{T}$,

$$
\operatorname{Mod}_{\mathfrak{T}}(\alpha) \preceq_{\mathfrak{T}} \operatorname{Mod}_{\mathfrak{T}}(\beta) .
$$

While truth is obviously dependent on the choice of a truth perspective $\mathfrak{T}$, one can prove that the notion of logical consequence represents an absolute relation that is invariant with respect to truth-perspective changes.

Theorem 8 [4]

$\alpha \vDash \beta$ iff for any model $\operatorname{Mod}, \operatorname{Mod}_{\mathrm{I}}(\alpha) \preceq_{\mathrm{I}} \operatorname{Mod}_{\mathrm{I}}(\beta)$, where I is the canonical truth-perspective.

On this basis one can conclude that:

- Alice and Bob may have different ideas about the logical connectives, about truth, falsity and probability.

- In spite of these differences, the reasoning-rules (which are determined by the logical consequence relation) are the same for Alice and for Bob. Apparently, assigning the same interpretation to the logical connectives is not a necessary condition in order to use the same reasoning-rules.

Acknowledgements We would like to thank Enrico Beltrametti, Maria Luisa Dalla Chiara and Roberto Giuntini, who have deeply discussed this paper with us, proposing some useful suggestions. Sergioli's work has been supported by the Italian Ministry of Scientific Research within the FIRB project "Structures and dynamics of knowledge and cognition", Cagliari unit F21J12000140001 and by Regione Sardegna within the project "Modeling the uncertainty: Quantum Theory and Imaging Processing"; Leporini's work has been supported by the Italian Ministry of Scientific Research within the PRIN project "Automata and Formal Languages: Mathematical Aspects and Applications".

\section{References}

1. E. Beltrametti, M.L. Dalla Chiara, R. Giuntini, R. Leporini, G. Sergioli, "Epistemic Quantum Computational Structures in a Hilbert-Space Environment", Fundamenta Informaticae 115, pp. 1-14, 2012. DOI 10.3233/FI-2012-637.

2. E. Beltrametti, M.L. Dalla Chiara, R. Giuntini, R. Leporini, G. Sergioli, "A Quantum Computational Semantics for Epistemic Logical Operators. Part I: Epistemic Structures", International Journal of Theoretical Physics 53 (10), pp. 3279-3292, 2014. 
3. E. Beltrametti, M.L. Dalla Chiara, R. Giuntini, R. Leporini, G. Sergioli, "A Quantum Computational Semantics for Epistemic Logical Operators. Part II: Semantics", International Journal of Theoretical Physics 53 (10), pp. 3293-3307, 2014.

4. E. Beltrametti, M.L. Dalla Chiara, R. Giuntini, G. Sergioli, "Quantum teleportation and quantum epistemic semantics", Mathematica Slovacca 62 (6), pp. 1-24, 2012.

5. G. Chiribella, G. M. D'Ariano, P. Perinotti, "Transforming quantum operations: Quantum supermaps", A Letters Journal Exploring the Frontiers of Physics 83, pp.30004p1-30004-p6, 2008.

6. M. L. Dalla Chiara, R. Giuntini, R. Leporini, "Logics from quantum computation", International Journal of Quantum Information 3, pp. 293-337, 2005.

7. M.L. Dalla Chiara, R. Giuntini, R.Leporini, G. Sergioli, "A first-order epistemic quantum computational semantics with relativistic-like epistemic effects", submitted.

8. Hong-yi Fan, Li-yun Hu, "Infinite-dimensional Kraus operators for describing amplitude-damping channel and laser process", Optics Communications 282, pp. 932935, 2009.

9. K. Kraus, States, Effects and Operations, Springer, Berlin, 1983. 